\title{
Anabases
}

ANABASES Traditions et réceptions de l'Antiquité

\section{Peter Liddel \& Polly Low (éd.), Inscriptions and their uses in Greek and Latin literature}

\section{Francesca Dell'Oro}

\section{(2) OpenEdition}

1 Journals

Édition électronique

URL : http://journals.openedition.org/anabases/5778

DOI : 10.4000/anabases.5778

ISSN : 2256-9421

Éditeur

E.R.A.S.M.E.

\section{Édition imprimée}

Date de publication : 10 novembre 2016

Pagination : 356-357

ISSN : 1774-4296

\section{Référence électronique}

Francesca Dell'Oro, « Peter Liddel \& Polly Low (éd.), Inscriptions and their uses in Greek and Latin

literature », Anabases [En ligne], 24 | 2016, mis en ligne le 15 novembre 2016, consulté le 24 septembre 2020. URL : http://journals.openedition.org/anabases/5778; DOI : https://doi.org/10.4000/anabases. 5778

Ce document a été généré automatiquement le 24 septembre 2020

(c) Anabases 


\title{
Peter Liddel \& Polly Low (éd.), Inscriptions and their uses in Greek and Latin literature
}

\author{
Francesca Dell'Oro
}

\section{RÉFÉRENCE}

Peter Liddel \& Polly Low (éd.), Inscriptions and their uses in Greek and Latin literature, Oxford, Oxford University Press, Oxford Studies in Ancient Documents, 2013, xii + $403 \mathrm{p}$. 94 livres / 978-0-19-966574-7

1 Dans ce volume, issu d'un colloque éponyme qui a eu lieu à l'Université de Manchester en 2009, P. Liddel et P. Low explorent la réception des inscriptions chez les auteurs grecs et latins. Dans une ample introduction ils explicitent les prémisses du colloque et de leur démarche scientifique en les inscrivant dans un projet de recueil et d'analyse de données épigraphiques transmises par les textes anciens, qu'ils ont commencé en 2003 en vue de la constitution du Database of inscriptions in Greek and Latin texts.

2 La première partie du recueil, caractérisée par une approche historiographique, est consacrée au dialogue que les auteurs anciens ont construit avec le passé. A. Hartmann montre comment le degré de fiabilité d'un document se conjuguait avec l'estimation de son ancienneté et $\mathrm{D}$. Langslow comment l'évolution de la langue et de l'écriture ont rendu difficiles la lecture et l'interprétation des inscriptions latines archaïques pour le public des époques postérieures. Les contributeurs soulignent en outre l'importance des sanctuaires et de leurs archives en tant que « centres de documentation » dans des genres variés, de l'histoire (E. Kosmetatou) à l'oratoire et à la philosophie (M. Mari) et ils retracent un éventail de modalités d'appropriation de la source que constituent les inscriptions, qui va de l'emploi d'un document original jusqu'à son invention, en passant par la révision et l'adaptation (M. Haake). L'épigramme inscriptionnelle est au centre de deux dernières contributions de cette section. Y.Z. Tzifopoulos se penche sur 
les modalités de la construction et de l'articulation de la narration autour des épigrammes dans l'œuvre de Pausanias. A. Petrovic, dans son étude sur la consultation et l'emploi par les orateurs athéniens du IV e siècle av. n. è. de collections d'épigrammes inscrites, propose la naissance d'une nouvelle typologie de collection épigrammatique qui aurait inclus les épigrammes publiques d'intérêt historique.

Dans la deuxième partie du volume les différentes études de cas dégagent non seulement les indices d'une réception de formules et motifs des inscriptions (en particulier métriques) chez les auteurs grecs et latins, mais elles montrent aussi que la pleine appréciation de cette réception devait passer par un public familier avec les codes épigraphiques. Aussi cette section contribue à l'exploration de l'« epigraphic literacy " - selon l'expression de J. Day (p. 217) - dans l'Antiquité gréco-romaine, comme le font D. Fearn à travers la poésie de Simonide et de Pindare, P. Le Ven avec l'exemple de l'hymne à la Vertu d'Aristote ou J. Nelis-Clément et D.P. Nelis, en présentant l'essor de la culture épigraphique sous Auguste. On remarquera que J. Lougovaya, en examinant la présence et la fonction des inscriptions dans les textes théâtraux, étend le sujet d'étude à un corpus qui était resté marginal. Novatrice apparaît aussi la contribution de M. Dinter qui exploite le cadre théorique élaboré par les études sur l'intermédialité afin d'analyser la perte des confins entre modalités inscriptionnelles et modalités narratives à l'instar de l'emploi de la formule d'origine épigraphique tu quoque dans l'Énéide. Le volume est clos par la contribution de A.V. Zadorojnyi, qui étudie la réception des inscriptions dans la prose d'âge impérial à l'exemple de plusieurs auteurs, de Dion de Pruse jusqu'au Roman d'Alexandre.

4 Trois index (inscriptions; textes littéraires et papyrus; sujets) enrichissent le volume et en permettent une consultation aisée pour un public aux intérêts diversifiés. Le recueil se caractérise par une typographie soignée, coquilles et omissions étant très limitées.

5 On aurait souhaité voir certains concepts de base mieux approfondis, comme, par exemple, ce qu'était une inscription pour les anciens, voire dans quelle mesure le support matériel influençait formes et contenus. Seuls quelques auteurs (par ex. Lougovaya, p. 255) affirment explicitement prendre en considération seulement les inscriptions exposées au public. Ce choix reflète une approche parfois difficile à mettre en œuvre lorsqu'un texte est présenté (cité, résumé ou employé) dans un autre texte, et la sélection des informations se trouve déjà faite par l'auteur ancien. Ambigu, bien que très courant, est en outre le terme d'« épigraphie littéraire » employé pour renvoyer à la réception des inscriptions chez les auteurs anciens, car ce syntagme finit, d'un côté, par qualifier de «littéraires» des documents qui peuvent avoir une composante «littéraire " très faible et qui n'ont souvent aucune prétention esthétique (par exemple, une borne qui marque une limite de propriété), et de l'autre, par exclure implicitement de l'étude spécifiquement littéraire certaines typologies d'inscriptions à cause de leur support (par exemple, les inscriptions métriques).

P. Liddel et $P$. Low offrent un volume dense et qui garde une cohérence polyphonique en dépit de l'ampleur du sujet choisi et de l'étendue chronologique du corpus. Il a, entre autres, le mérite de réunir les contributions de spécialistes provenant de champs disciplinaires aux approches et aux méthodes différentes, comme le sont l'histoire et la littérature. Ce volume est sans doute destiné à être un point de départ utile pour d'ultérieurs approfondissements des rapports entre épigraphie et littérature. 


\section{AUTEURS}

FRANCESCA DELL'ORO

Chercheuse indépendante

francesca.delloro@unil.ch 